Document downloaded from:

http://hdl.handle.net/10251/82026

This paper must be cited as:

Guarnizo, JG.; Mellado Arteche, M. (2016). Robot Soccer Strategy Based on Hierarchical Finite State Machine to Centralized Architectures. IEEE Latin America Transactions. 14(8):3586-3596. doi:10.1109/TLA.2016.7786338.

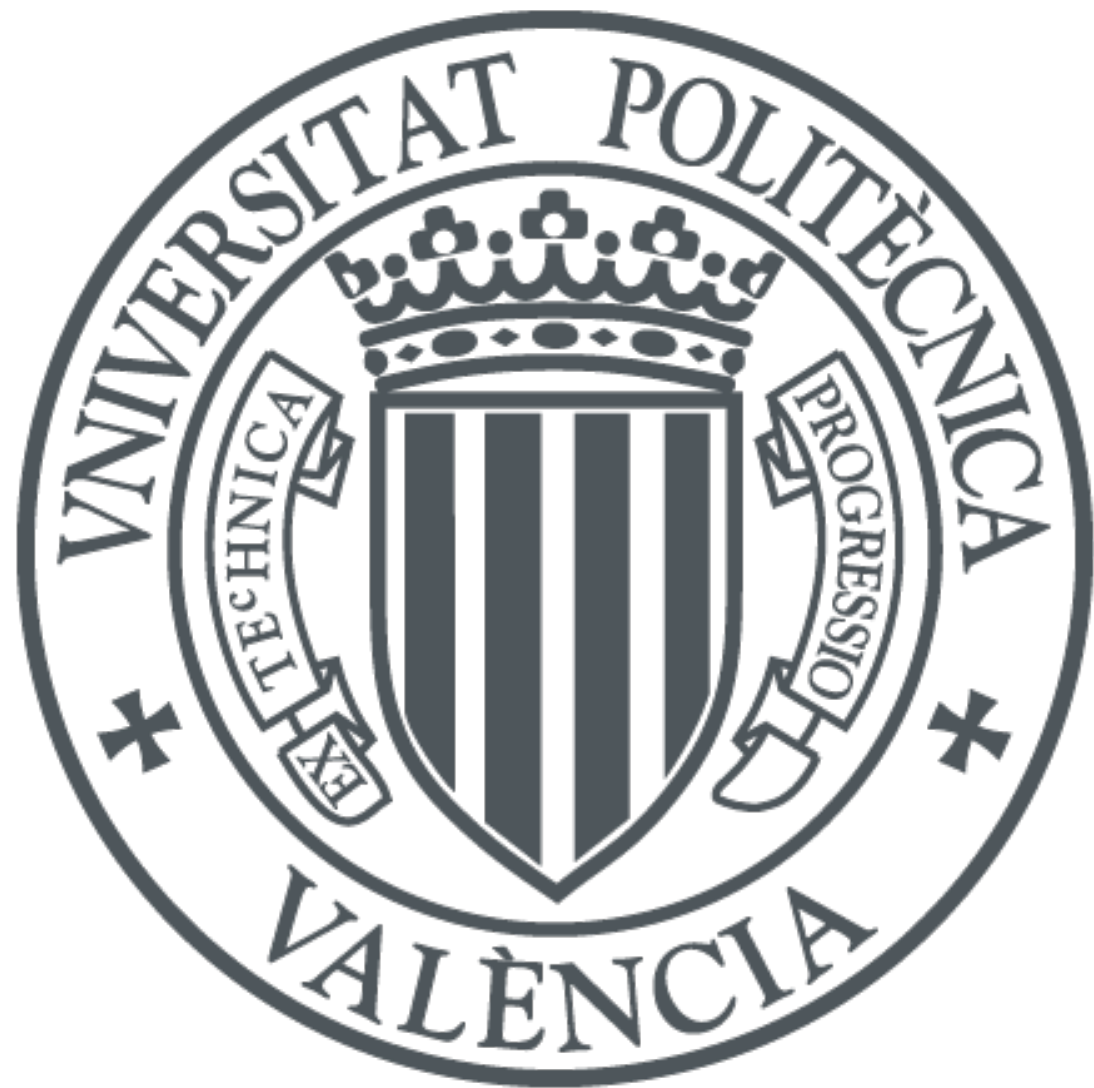

The final publication is available at

http://ieeexplore.ieee.org/document/7786338/

Copyright Institute of Electrical and Electronics Engineers (IEEE)

Additional Information

(C) 2016 IEEE. Personal use of this material is permitted. Permission from IEEE must be obtained for all other uses, in any current or future media, including reprinting/republishing this material for advertising or promotional purposes, creating new collective works, for resale or redistribution to servers or lists, or reuse of any copyrighted component of this work in other works 


\title{
Robot Soccer Strategy Based on Hierarchical Finite State Machine to Centralized Architectures
}

\author{
J. G. Guarnizo and M. Mellado
}

\begin{abstract}
Coordination among the robots allows a robot soccer team to perform better through coordinated behaviors. This requires that team strategy is designed in line with the conditions of the game. This paper presents the architecture for robot soccer team coordination, involving the dynamic assignment of roles among the players. This strategy is divided into tactics, which are selected by a Hierarchical State Machine. Once a tactic has been selected, it is assigned roles to players, depending on the game conditions. Each role performs defined behaviors selected by the Hierarchical State Machine. To carry out the behaviors, robots are controlled by the lowest level of the Hierarchical State Machine. The architecture proposed is designed for robot soccer teams with a central decision-making body, with global perception. 200 games were performed against a team with constant roles, winning the $92.5 \%$ of the games, scoring more goals on average that the opponent, and showing a higher percent of ball possession. Student's t-test shows better matching with measurement uncertainty of the strategy proposed. This architecture allowed an intuitive design of the robot soccer strategy, facilitating the design of the rules for role selection and behaviors performed by the players, depending on the game conditions. Collaborative behaviors and uniformity within the players' behaviors during the tactics and behaviors transitions were observed.
\end{abstract}

Keywords - Robot soccer, strategy, multi-robot systems, architecture, coordination.

\section{INTRODUCCIÓN}

$\mathrm{S}$ ISTEMAS multi-agente presentan una alternativa para resolver problemas de sistemas que necesitan trabajar en conjunto con el fin de alcanzar un objetivo específico [1]. El fútbol de robots presenta como un entorno multi-agente en un ambiente hostil con incertidumbres, donde los robots deben operar en la búsqueda de un objetivo común [2]. En fútbol de robots la estrategia es definida como el plan del equipo con el que se pretende ganar el partido, la táctica se define como la organización del equipo durante un momento dado [3]. Los roles corresponden a la combinación de los comportamientos de los robots junto a su ubicación en el campo de juego, por ejemplo portero, mediocampista, defensa o delantero. Los comportamientos corresponden a los movimientos que realizan los robots con el fin de realizar tareas específicas, como ir por el balón, bloquear un oponente o realizar un pase [4]. Los comportamientos también son utilizados en robots

J. G. Guarnizo, Laboratorio de Investigación en Fuentes Alternativas de Energía LIFAE, Universidad Distrital Francisco José de Caldas, jguarnizo@correo.udistrital.edu.co

M. Mellado, Instituto de Automática e Informática Industrial, Universitat Politècnica de València, martin@ai2.upv.es móviles para esquemas de control de navegación [5].

En este artículo se presenta una arquitectura de equipo de fútbol de robots basada en una Máquina Jerárquica de Estado Finito para selección de tácticas, comportamientos y acciones de control a bajo nivel, envolviendo el concepto de un "técnico virtual" para el proceso de asignación de roles. La táctica es seleccionada por la Máquina de Estado dependiendo de la posición del balón y los jugadores. Posteriormente los roles son asignados a los jugadores dependiendo de su posición en el campo de juego, siendo este proceso realizado por un agente virtual llamado técnico. Posteriormente la Máquina de Estado selecciona los comportamientos de los jugadores, dependiendo de la táctica y su rol. La propuesta presenta como ventajas una sencilla programación e implementación, sin mayor complejidad y fácilmente adaptable a cualquier clase de lenguaje de programación. Así mismo permite actualizar la estrategia con nuevos cambios o ampliarla a más jugadores sin ajustes significativos. Con esto se busca presentar un diseño de estrategia intuitivo, fácilmente adaptable, sin que requiera excesiva complejidad en el momento de su programación e implementación.

Esta arquitectura es diseñada para equipos de fútbol de robots con un sistema central de toma de decisiones y percepción global, simulada utilizando robots diferenciales de dos ruedas mediante el uso del simulador Webots. En el capítulo II se presentan los trabajos relacionados. En el capítulo III se presenta la arquitectura. En el capítulo IV se presenta la validación y resultados. En el capítulo V las conclusiones son detalladas.

\section{TRABAJOS RELACIONADOS}

En fútbol de robots se presentan ligas con arquitecturas que utilizan robots autónomos con percepción local y control distribuido (en adelante llamadas ligas distribuidas), o ligas con arquitecturas con percepción global y control centralizado (en adelante ligas centralizadas). Dependiendo del tipo de liga, los trabajos de coordinación de equipo requerirán de consideraciones diferentes. En el caso de las ligas distribuidas se observan arquitecturas de coordinación de equipo que envuelven el concepto de formación de jugadores [6]. Otras trabajan funciones heurísticas y deliberación para selección de roles [7]. Otros trabajos plantean arquitecturas modulares y jerárquicas basadas en módulos paralelos y capas [8]. Otras arquitecturas utilizan una técnica para el diseño mecatrónico de sistemas [9]. Redes de Petri son también utilizadas en equipos de fútbol de robots [10]. Otros trabajos se enfocan en el diseño de sistemas de toma de decisiones, utilizando 
gráficos de coordinación para asignación de roles [11], Máquinas de Estado Finito para la selección de tácticas [12] o para la selección de comportamientos en los robots [13].

Arquitecturas de coordinación de equipo para ligas centralizadas presentan importantes diferencias con respecto a las arquitecturas diseñadas para ligas distribuidas, por lo que requiere de criterios de diseño diferentes. Se tiene por ejemplo Sistemas de Eventos Discretos para la selección de comportamientos [14]. En [15] presentan asignación dinámica de roles por medio de teoría de juegos, la táctica es seleccionada dependiendo de una función de utilidad que tiene en cuenta la información del equipo. Máquinas Jerárquicas de Estado Finito son utilizadas en [16] para la selección de tácticas, asignación de roles y tareas. En [17] se proponen estrategias que dividen el campo de juego en cuadrículas con el fin de crear vectores de posición de los jugadores y el balón, donde a partir de un vector actual de posiciones se crea un vector de posiciones deseado para los jugadores. En [18] utilizan un sistema de toma de decisiones auto adaptativo en la asignación de roles y tareas, a partir de los modelos matemáticos de las misiones que los robots deben desarrollar. Aprendizaje de sistemas de toma de decisiones mediante técnicas Q-Learning también es utilizado en [19] para la selección de estrategias y roles. En [20] combinan holones con Sistemas Inmunes Artificiales para el diseño de una arquitectura jerárquica. En [21] Árboles de Decisión son utilizados para selección de roles. Otros trabajos se enfocan en el diseño de comportamientos de agentes, por ejemplo en [22] dos agentes colaboran en la realización de un pase utilizando Redes Neuronales. Diagramas de Voronoy se presentan en [23] para coordinar pases entre jugadores. En [24] utilizan Árboles de Decisión para la coordinación de pases.

Lógica Difusa es utilizada para determinar qué jugador se encarga de un área de juego determinada en caso de conflicto [25], o para obtener parámetros que son usados por algoritmos Q-Learning para calcular las trayectorias de los jugadores [26]. En [27] se usa un mecanismo difuso para seleccionar comportamientos de un robot a través de la posición de los jugadores, validado únicamente en juegos 1vs.1 sin envolver coordinación. Sistemas de inferencia difusos son las entradas de una Máquina de Estado para la selección de comportamientos de un robot futbolista autónomo [28]. En [29] los valores de afinidad son fusificados para un sistema de toma de decisiones basado en Sistemas Inmunes Artificiales, para la selección de comportamientos de los jugadores. En [30] se utiliza una Red Neuro-Difusa para la planeación de trayectorias y el control de movimiento de los jugadores. En [31] proponen una arquitectura multi-nivel con Lógica Difusa para la planeación de trayectorias y el control de movimiento de los robots, aunque no se presenta una arquitectura global ni comportamientos colaborativos. Si bien la Lógica Difusa ha sido una herramienta en el fútbol de robots esta no se plantea como el sistema de toma de decisiones, sino que se utiliza para fusificar los valores de entrada de sistemas de toma de decisiones que utilizan diferentes modelos computacionales, o para el control de movimientos y la planeación de trayectorias de los robots.
Redes Bayesianas se utilizan también para la coordinación de equipo en fútbol de robots, con el fin de estimar el comportamiento de un jugador entre disparar o driblar el balón en equipos distribuidos [32], o como entrada de una Red Neuronal para la selección de la estrategia en un equipo de arquitectura centralizada [33]. Estos trabajos requieren el aprendizaje de las reglas de la red Bayesiana, siendo la misma parte del sistema de toma de decisiones, combinada con otros modelos computacionales.

Si bien han sido presentados distintos trabajos con fines de coordinación de equipo, estos presentan complejos algoritmos para su diseño haciendo difícil su adaptación a nuevos jugadores, nuevas ligas o nuevos reglamentos o se enfocan en el diseño de algoritmos de aprendizaje sin centrarse en la arquitectura de equipo. En otro caso diseñan comportamientos de roles o jugadas muy específicas. Además muchos se enfocan en ligas distribuidas, las cuales como se dijo presentan un paradigma diferente.

\section{ARQUITECTURA JERÁRQUICA}

Como se ha mencionado anteriormente, las Máquinas de Estado son frecuentemente utilizadas en fútbol de robots como alternativas para el diseño de estrategias de equipo. En este capítulo se propone un sistema de toma de decisiones basada en una Máquina Jerárquica de Estado Finito, la cual es dividida en distintos niveles. El primer nivel es presentando en la Fig. 1, donde una Máquina de Estado selecciona la táctica desde un conjunto de $n$ predefinidas tácticas $T=\left\{t_{1}, t_{2}, \ldots, t_{n}\right\}$, utilizando las condiciones ambientales $E v$ como transiciones.

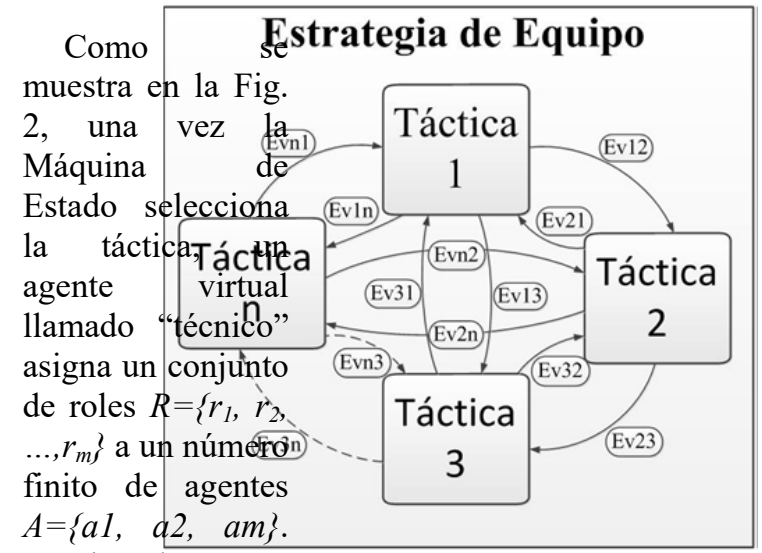

LФ\$்y ytagladoiresr cionl para el sistema de toma de decisiones. sus respectivos

roles ejecutan un comportamiento de un conjunto de comportamientos $B=\left\{b_{1}, b_{2}, \ldots, b_{k}\right\}$, dicho comportamiento es seleccionado por el segundo nivel de la Máquina de Estado utilizando las condiciones ambientales $S t$ como transiciones. El control de los comportamientos es realizado por la capa más baja de la Máquina de Estado.

El técnico mide las condiciones ambientales de forma periódica, cuando estas condiciones cambian, una nueva táctica es seleccionada y un nuevo conjunto de roles es asignado a los jugadores. Un diagrama del modelo propuesto es presentado en la Fig. 3. 


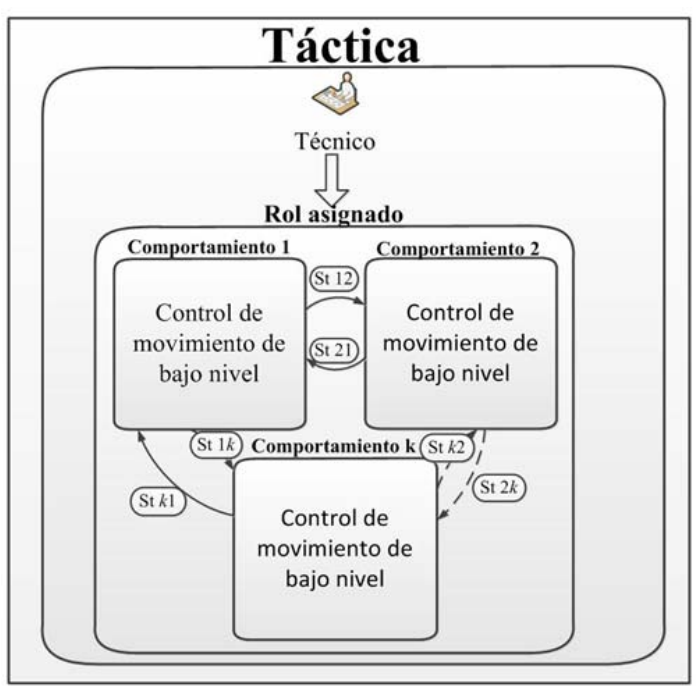

Figura 2. Niveles de asignación de roles y selección de comportamientos.

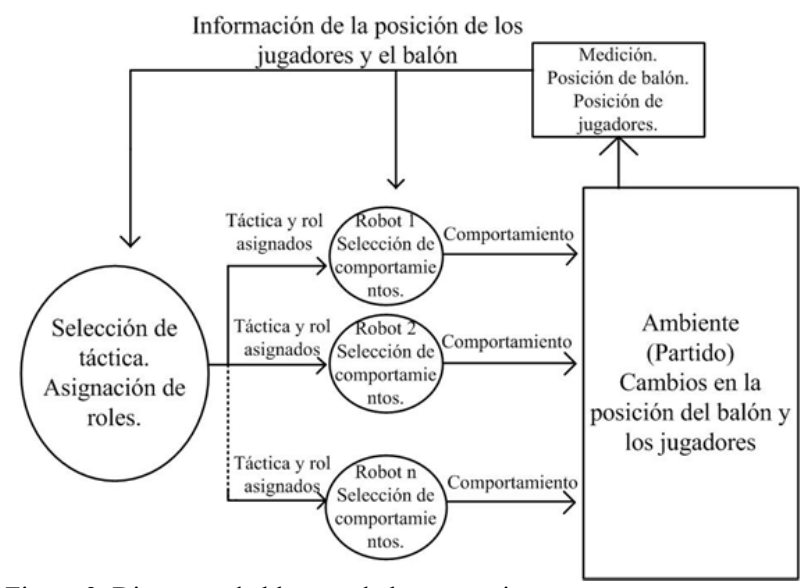

Figgara 3. Diagrama de bloques de la estrategia.

asignación de

táctica es realizada cuando ocurre el evento que genera la transición correspondiente en la Máquina de Estado. No obstante si las condiciones de juego no han cambiado considerablemente los roles deben mantenerse y los comportamientos desarrollados por los jugadores no deben ser interrumpidos o cambiados de manera abrupta perdiendo la continuidad del juego. El objetivo de la estrategia propuesta es asegurar un adecuado rendimiento buscando ganar el partido. Esta estrategia es implementada en un equipo de fútbol de robots con 4 jugadores. Uno de ellos es el portero, el cual es el único jugador con rol constante. Para la selección de tácticas se seleccionaron dos parámetros: el primero corresponde a la localización del balón en el campo de juego y el segundo a la posesión del balón. El campo se ha dividido en 3 zonas como se presenta en la Fig. 4. (Todas estas imágenes han sido directamente tomadas del simulador Webots), con el fin de clasificar la localización del balón en una de estas zonas.

Estas zonas han sido divididas como se muestra a continuación. Dada una distancia $l$ que corresponde al largo del campo de juego, siendo esta la distancia entre la portería propia y la portería del equipo oponente, $l$ es dividido en dos distancias menores $l / m$ y $l / n$ tal que $0<l / n<l / 2$ y $1 / 2<l / m<$ $l$, donde $n \in \mathcal{R}, m \in \mathcal{R}$, y $n>m$. Dado esto, se define:

$$
l_{b} \in \mathcal{R}>0 \text {, tal que } l_{b} \leq l
$$

Donde $l_{b}$ es la distancia entre la portería propia y el balón. Se define $Z z$ como la zona del campo de juego donde el balón está ubicado.

Dado esto las tres zonas se definen de la siguiente manera:

- Zona defensiva: $Z_{Z}=Z_{D}: \exists l_{b}$ tal que $l_{b} \leq l / n$.

- Zona media: $Z_{Z}=Z_{M}: \exists l_{b}$ tal que $l / n<l_{b} \leq l / m$.

- Zona ofensiva: $Z_{Z}=Z_{M}$ : $\exists l_{b}$ tal que $l / m<l_{b} \leq l$.

$n$ y $m$ determinan la ubicación de las zonas a lo largo del campo de juego, con la posibilidad de adaptar estos valores en caso que se necesite una táctica más defensiva u ofensiva. Se define $R a_{m}$ como el vector de coordenadas $(x m(t), y m(t))$ de un agente am sobre el campo de juego, tal que

$$
x m(t) \in \mathcal{R} \text { tal que } 0 \leq x m(t) \leq l,
$$

$y m(t) \in \mathcal{R}$ tal que $0 \leq y m(t) \leq w$,

Donde $w$ corresponde al ancho del campo de juego. Se define $R B a l_{b}$ como el vector de las coordenadas del balón sobre el campo de juego, tal que:

$$
\begin{aligned}
& x_{b}(t) \in \mathcal{R} \text { tal que } 0 \leq x_{b}(t) \leq l, \\
& y_{b}(t) \in \mathcal{R} \text { tal que } 0 \leq y_{b}(t) \leq w,
\end{aligned}
$$

Dado lo anterior, se tiene $D\left(R B a l_{b}, R a_{m}\right)$, que corresponde a la distancia euclídea entre $a_{m}$ y el balón:

$$
D\left(R B a l_{b}, R a_{m}\right)=\left\|\left(x_{b}(t)-x m(t), y b(t)-y m(t)\right)\right\|_{2},
$$

Teniendo en cuenta el sistema de coordenadas utilizado en arquitecturas con percepción global, es posible modelar el campo de juego como un espacio euclidiano, lo que permite obtener la distancia euclidiana entre dos puntos como la mínima distancia entre los mismos.

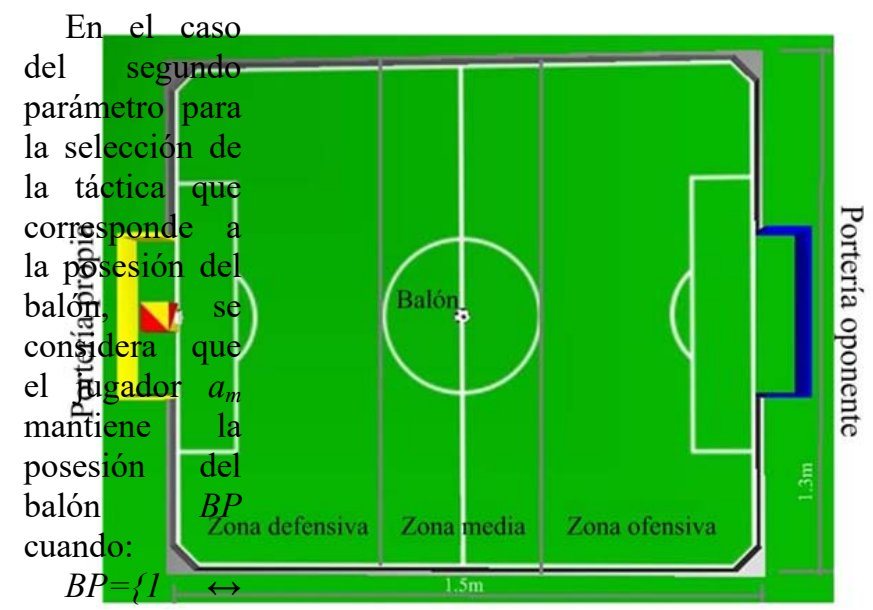

$D$ PRPBREl

$\leq \delta_{b}$,

$$
\left.0 \leftrightarrow D\left(R B a l l, R a_{m}\right)>\delta_{b}\right\},
$$

Donde $\delta_{b}$ es la máxima distancia en la que se considera que el robot tiene posesión del balón $y$ corresponde a la distancia máxima en la que el robot puede golpear el balón. Para calcular este valor es necesario tener en cuenta que las coordenadas de la posición tanto del robot como del balón son calculadas a partir de sus respectivos centros de gravedad en el plano $(x, y)$. Dado esto se obtiene la máxima distancia en la que el robot al realizar un giro sobre su propio eje hace contacto con el balón y lo envía en la dirección donde fue realizado el giro, considerando esta distancia como la máxima en la que un robot tiene posesión del balón. Para esta 
estrategia fueron asignados los siguientes valores: $l=1.5 \mathrm{~m}$, $w=1.3 \mathrm{~m}, \delta_{b}=0.008 \mathrm{~m}$, donde $l \mathrm{y} w$ corresponden a las dimensiones del campo de juego tomadas directamente del simulador Webots.

Un número finito de tácticas $T=\left\{t_{d d}, t_{d a}, t_{o d}, t_{o a}\right\}$ ha sido definido a continuación.

Táctica defensa $\left(T_{d d}=1\right)$ se selecciona cuando el balón está localizado en la zona defensiva $\left(Z_{D}=1\right)$ o en la zona media $\left(Z_{M}=1\right)$, y ningún jugador del equipo propio tiene la posesión del balón $(-B P=1)$.

$\operatorname{Si}\left(\left(Z_{D}=1 \vee Z_{Z}=1\right) \wedge\ulcorner B P=1)\right.$,

entonces $T=t_{d d}$,

Táctica contra ataque $\left(T_{d a}=1\right)$ es seleccionada cuando el balón está ubicado en la zona defensiva $\left(Z_{D}=1\right)$ o en la zona media $\left(Z_{M}=1\right)$, y algún jugador del equipo propio tiene la posesión del balón $(B P=1)$.

Si $\left(\left(Z_{D}=1 \vee Z_{Z}=1\right) \wedge B P=1\right)$,

entonces $T=t_{d a}$

Táctica presión $\left(T_{o d}=1\right)$ es seleccionada cuando el balón está localizado en la zona ofensiva $\left(Z_{O}=1\right)$ y ningún jugador del equipo propio tiene la posesión del balón $(-B P=1)$.

Si $\left(Z_{O} \wedge-B P\right)$

entonces $T=t_{\text {od }}$,

Táctica ataque $\left(T_{o a}=1\right)$ es seleccionada cuando el balón se encuentra en la zona ofensiva $\left(Z_{O}=1\right)$ y algún jugador del equipo propio tiene la posesión del balón $(B P=1)$.

Si $\left(Z_{O} \wedge B P\right)$

entonces $T=t_{o a}$,

Las reglas de la selección de tácticas son presentadas en la Tabla I.

TABLA I

CRITERIOS DE SELECCIÓN DE TÁCTICA DEL PRIMER NIVEL DE LA MÁQUINA DE ESTADOS

\begin{tabular}{ccc}
\hline Táctica & $\begin{array}{c}\text { Zona de ubicación } \\
\text { del balón }\end{array}$ & $\begin{array}{c}\text { Posesión del } \\
\text { balón }\end{array}$ \\
\hline $\begin{array}{c}\text { Defensa } \\
\text { Contra }\end{array}$ & Defensiva y media & No \\
ataque & Defensiva y media & Sí \\
Presión & Ofensiva & No \\
c &
\end{tabular}

\section{A. Asignación de roles}

Una vez la táctica ha sido seleccionada, los roles se asignan dependiendo no solo de la táctica, sino también de la posición de los jugadores y el balón. Para esto Rga se define como las coordenadas del centro de la portería propia (xga,yga), tal que $x g a=0$ y $y g a=w / 2$.

$D\left(R g a, R a_{m}\right)$ se define como la distancia entre $a_{m}$ y $R g a$, como se presenta a continuación:

$D\left(R g a, R a_{m}\right)=\|(x g a-x m(t), y g a-y m(t))\|_{2}$,

Se define Rge como las coordenadas del centro de la portería oponente (xge, yge) tal que $x g e=l$ y $y g e=w / 2$. $D\left(R g e, R a_{m}\right)$ se define como la distancia entre $a_{m}$ y $R g e$, como se presenta a continuación:

$D\left(R g e, R a_{m}\right)=\|(x g e-x m(t), y g e-y m(t))\|_{2}$,

Como se explicó anteriormente, hay 4 jugadores por equipo. Uno de ellos es el portero $a_{p}$, siendo el único rol constante y asignado al mismo jugador. Dado esto, existe un número finito de agentes $A=\left\{a_{1}, a_{2}, a_{3}\right\}$, que ejecutan un conjunto de roles $R=\left\{r_{d}, r_{s}, r_{a}\right\}$, donde $r_{d}$ corresponde al defensa, $r_{s}$ es el mediocampista y $r_{a}$ corresponde al delantero. El técnico $C$ ejecuta el algoritmo de asignación de roles, dependiendo de la táctica previamente seleccionada, de acuerdo con el siguiente procedimiento.

Táctica defensa: En esta táctica, el jugador más cercano al balón se convierte en el defensa $r_{d}$ y está encargado de tratar de capturar el balón. El jugador más cercano a la portería oponente es el delantero $r_{a}$, quien va a la mitad del campo de juego a la espera del balón para atacar. El último jugador es el mediocampista $r_{s}$, quien va a bloquear al jugador oponente más cercano a la portería propia, con propósitos de defensa y tratando de bloquear posibles pases del equipo rival. Un ejemplo de esta situación de juego se presenta en la Fig. 5. Para la asignación de roles, el técnico virtual $\mathrm{C}$ ejecuta el algoritmo 1 .

ALGORITMO 1. Asignación de roles, táctica defensa.

1. Obtener $\min \left\{D\left(R B a l_{b}, R a_{1}\right), D\left(R_{B a l l}, R a_{2}\right), D\left(R_{B a l l}\right.\right.$, $\left.\left.R a_{3}\right)\right\}$.

2. Asignar $a_{d}$ al agente con la menor distancia calculada en el paso 1.

3. Asignar $r_{d}=a_{d}$.

4. Obtener $\min \left\{D\left(R g e, R a_{1}\right), D\left(R g e, R a_{2}\right), D\left(R g e, R a_{3}\right)\right\}$, excluyendo

5. $D\left(\right.$ Rge,$\left.R a_{d}\right)$.

6. Asignar $a_{a}$ al agente con la menor distancia obtenida en el paso 4.

7. Asignar $r_{a}=a_{a}$

8. Seleccionar $a_{s}=\left\{\left\{a_{1}, a_{2}, a_{3}\right\}\right.$ tal que $\left.\left(a_{s} \neq a_{d}\right) \wedge\left(a_{s} \neq a_{a}\right)\right\}$

9. Asignar $r_{s}=a_{s}$.

Táctica contra ataque: Para asignar roles, el robot con la posesión del balón se convierte en el mediocampista $r_{s}$, quien enviará el balón a la zona ofensiva. El delantero $r_{a}$ es el jugador más
cercano
portería
oponente quien
debe esperar la
balón
enviará
mediocampista.
El otro jugador
es el defensa $r_{d}$,
quien
mantiene
esperando en la
zona defensiva

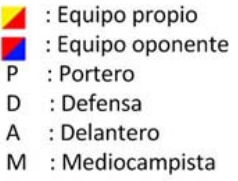

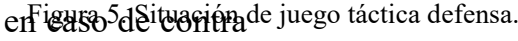

golpes. En el

momento que el delantero intercepte el balón, la Máquina de Estado activa una nueva táctica y un nuevo proceso de sección de roles es activado. Un ejemplo de situación de juego se presenta en la Fig. 6. Para la asignación de roles, el técnico virtual $\mathrm{C}$ ejecuta el algoritmo 2. 

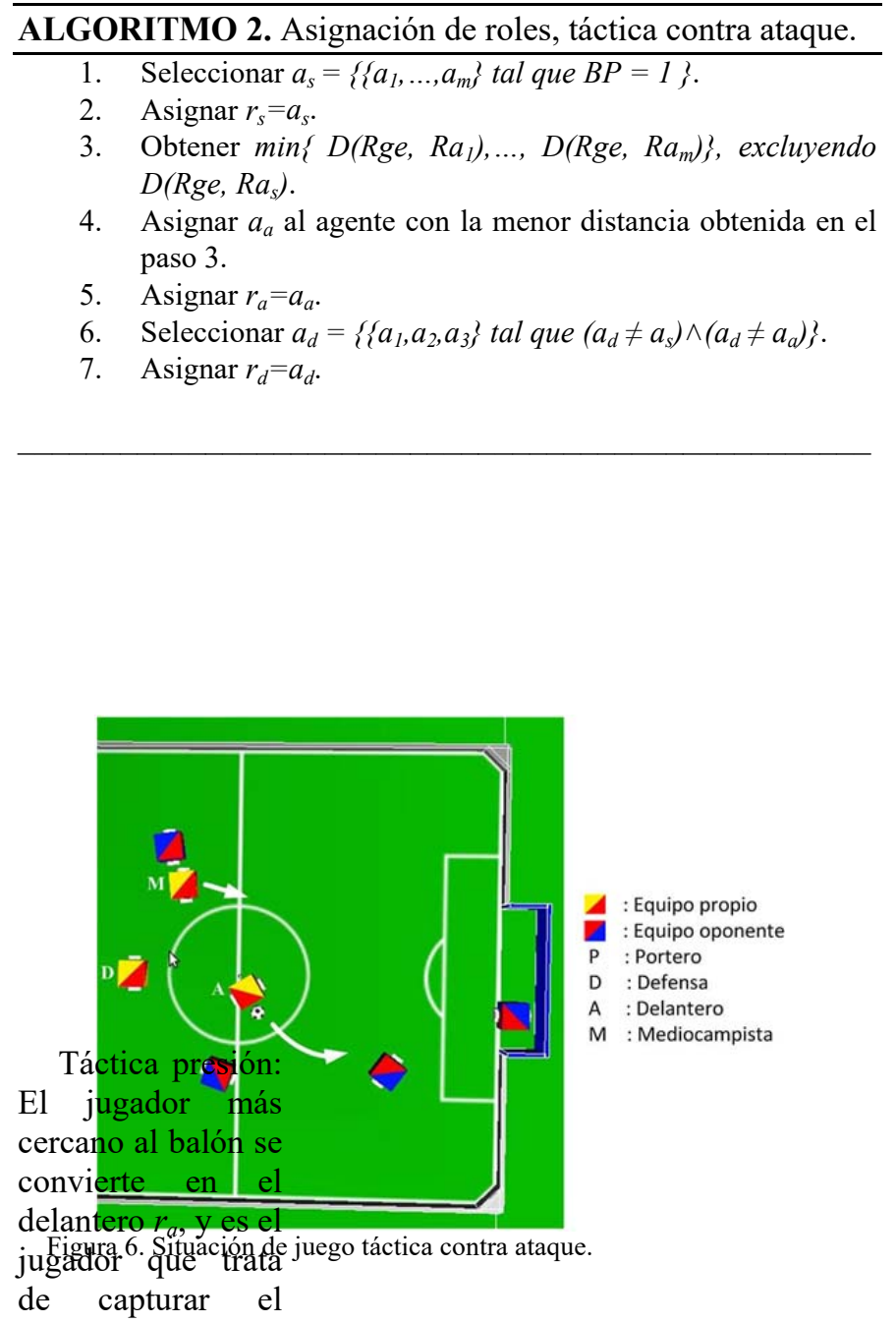

balón. El defensa $r_{d}$ es el jugador más cercano a la portería propia, y se mantiene en la zona defensiva en caso de contra ataque del equipo rival. El último es el mediocampista $r_{s}$, quien se encuentra bloqueando al jugador oponente más cercano. La Fig. 7 muestra un ejemplo de esta táctica, donde el delantero trata de interceptar el balón, el mediocampista bloquea un oponente y el defensa espera. Para la asignación de roles, el técnico virtual $\mathrm{C}$ ejecuta el algoritmo 3.

ALGORITMO 3. Asignación de roles, táctica presión.

1. Obtener $\min \left\{D\left(R_{B a l l}, R a_{1}\right), D\left(R_{B a l l}, R a_{2}\right), D\left(R_{B a l l}\right.\right.$, $\left.\left.R a_{3}\right)\right\}$.

2. Asignar $a_{a}$ al agente con la menor distancia calculada en el paso 1.

3. Asignar $r_{a}=a_{a}$.

4. Obtener $\min \left\{D\left(R g a, R a_{1}\right), D\left(R g a, R a_{2}\right), D\left(R g a, R a_{3}\right)\right\}$, excluyendo $D\left(R g a, R a_{a}\right)$.

5. Asignar $a_{d}$ al agente con la menor distancia obtenida en el paso 4.

6. Asignar $r_{d}=a_{d}$.

7. Seleccionar $a_{s}=\left\{\left\{a_{1}, a_{2}, a_{3}\right\}\right.$ such as $\left.\left(a_{s} \neq a_{d}\right) \wedge\left(a_{s} \neq a_{a}\right)\right\}$

8. Asignar $r_{s}=a_{s}$.

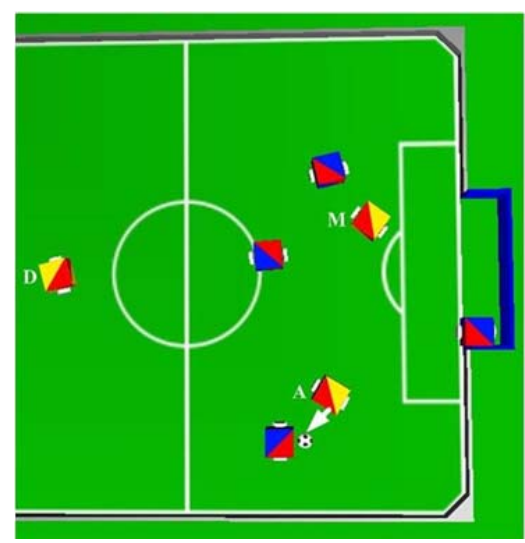

Figura 7. Situación de juego táctica presión.
Táctica

ataque: $\quad \mathrm{El}$ jugador con la posesión del balón se :EQupderfisenen el delandero $r_{a}$, ciufensamisión es tradalscahtsistanotar un gol. El jugador más cercano a la portería propia es el defensa $r_{d}$, quien espera en la zona defensiva en caso de contra ataque. El último jugador es el que obtiene el rol de mediocampista, ubicado en paralelo con el delantero en línea con la longitud del campo de juego y a la espera de recibir un posible pase o recuperar el balón. Un ejemplo de situación de juego se presenta en la Fig. 8(a), donde el delantero trata de anotar un gol. En caso que un jugador oponente diferente al portero bloquea la línea de gol a la portería rival, el delantero le envía el balón al mediocampista, como se presenta en la Fig. 8(b). Para la asignación de roles, el técnico virtual $\mathrm{C}$ ejecuta el algoritmo 4.

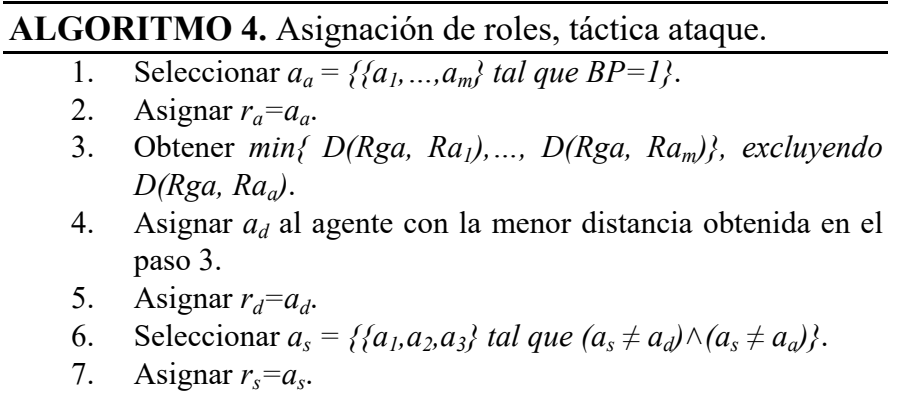

\section{B. Selección de comportamientos}

Diferentes roles han sido diseñados con el fin de ejecutar

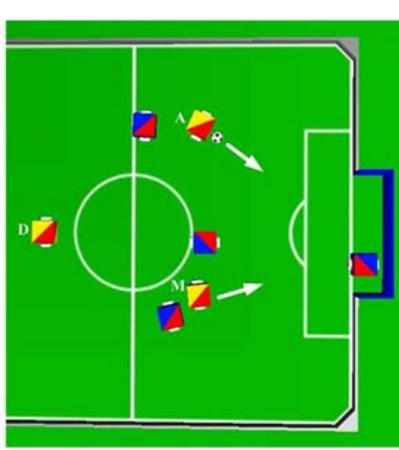

(a)

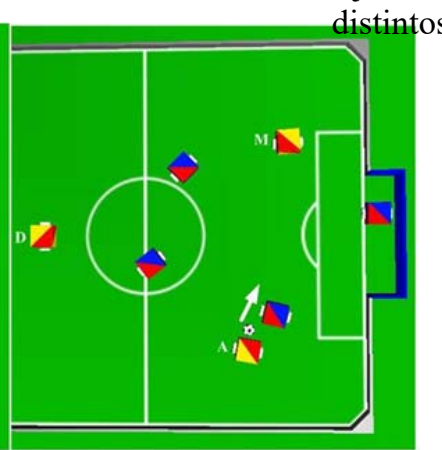

(b)

Figura 8. Situación de juego táctica ataque. 
comportamientos, dependiendo de la táctica seleccionada. Como se puede observar en la Tabla II, cada rol en cada táctica posee un comportamiento principal. Para el caso del portero, se ha determinado un único rol que consiste en seguir el balón en paralelo a la línea de gol.

TABLA II

COMPORTAMIENTOS PRINCIPALES DE LOS ROLES PARA CADA TÁCTICA

\begin{tabular}{lll}
\hline Táctica & \multicolumn{1}{c}{ Rol } & \multicolumn{1}{c}{ Comportamiento principal } \\
\hline Defensa & Defensa & Ir por el balón \\
& Mediocampista & Bloquear jugador oponente \\
& Delantero & Ir a la mitad del campo de juego \\
Contra & Defensa & Bloquear jugador oponente \\
ataque & Mediocampista & Enviar balón a zona ofensiva \\
& Delantero & Esperar pase \\
Presión & Defensa & Ir a zona defensiva \\
& Mediocampista & Bloquea jugador oponente \\
& Delantero & Ir por el balón \\
Ataque & Defensa & Ir a zona defensiva \\
& Mediocampista & Espera pase \\
& Delantero & Realizar pase o disparar a la
\end{tabular}

Los comportamientos principales se resumen a continuación.

$\checkmark$ Ir por el balón: El robot va hacia el balón hasta la distancia donde se considera que tiene posesión del mismo $(0.08 \mathrm{~m})$.

$\checkmark$ Bloquear jugador oponente: Identificar un jugador oponente determinado, y bloquearlo sin tener contacto físico con el mismo.

$\checkmark \quad$ Ir a la mitad del campo de juego: Ir a la mitad del campo de juego, esperando por el balón en caso de contra ataque.

$\checkmark \quad$ Enviar balón a zona ofensiva: Disparar el balón hacia la zona ofensiva del campo de juego.

$\checkmark$ Esperar pase: Desde una posición determinada, esperar que jugador compañero envíe un pase.

$\checkmark \quad$ Ir a zona defensiva: Ir a la zona defensiva, esperando en caso de contra ataque del equipo oponente.

$\checkmark$ Disparar a la portería o Realizar pase: Disparar el balón a la portería oponente, tratando de anotar un gol, en caso de que la línea de gol no esté bloqueada por un oponente diferente al portero. O enviar el balón a otro robot, cuando la línea de gol de la portería oponente está bloqueada por otro jugador oponente diferente al portero.

Los comportamientos principales se dividen en subcomportamientos, seleccionados por el segundo nivel de la Máquina Jerárquica de Estado Finito. En la Fig. 9 se muestran los estados de los comportamientos principales y los subcomportamientos. En el Anexo es presentado el pseudocódigo de la estrategia global presentada en este capítulo.

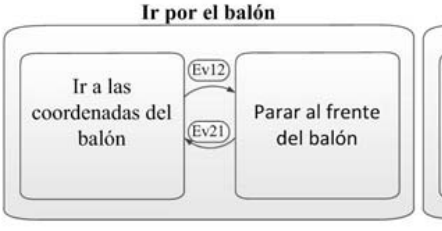

Bloquear jugador oponente

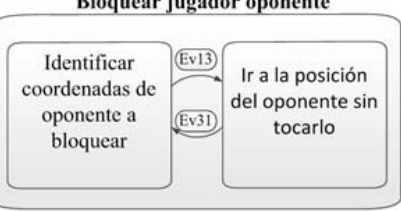

Ir a la mitad del campo de juego

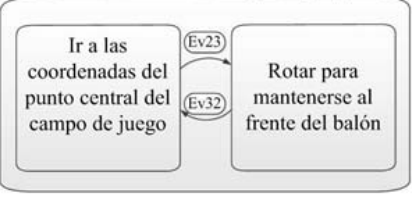

Enviar el balón a zona ofensiva
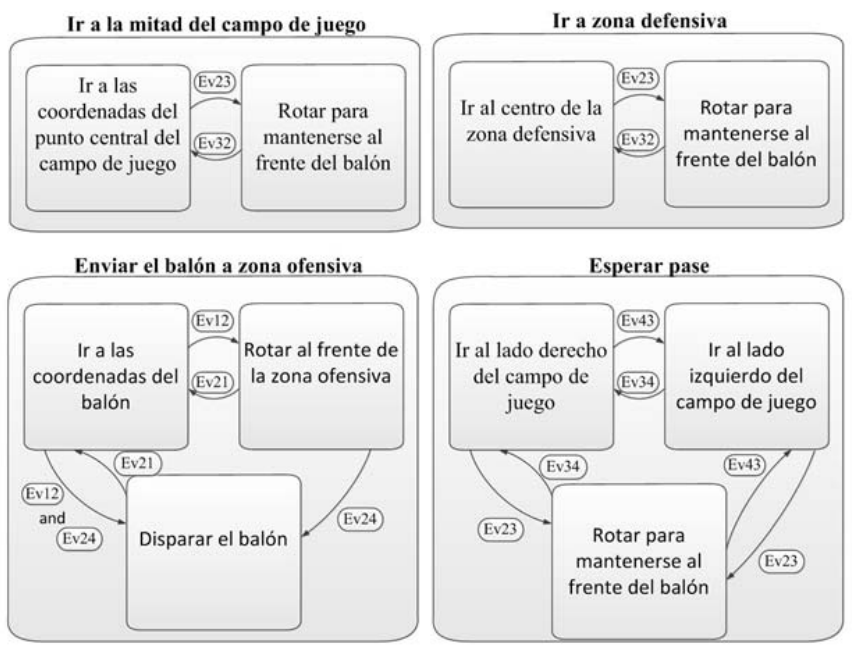

Enviar un pase o disparar a la portería

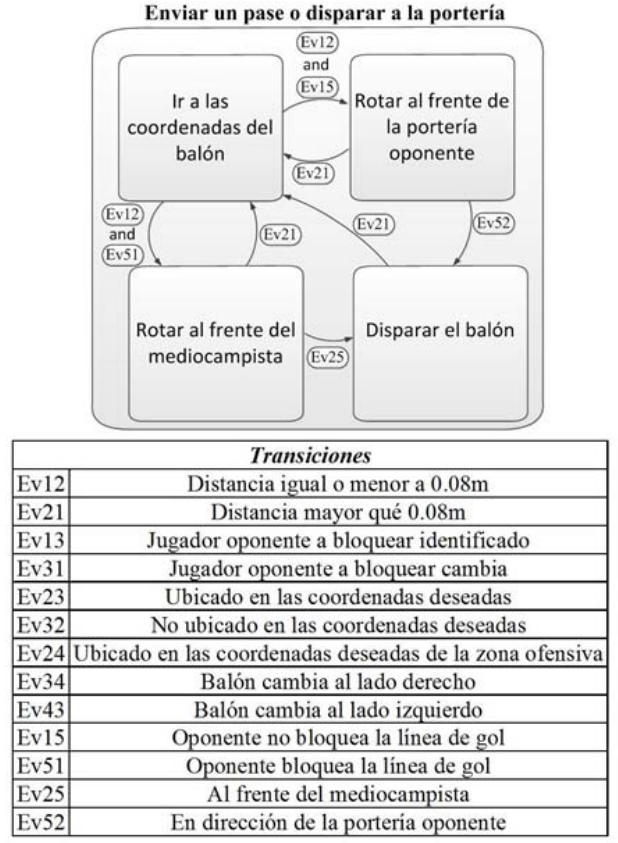

Figura 9. Comportamientos y transiciones.

\section{VALIDACIÓN Y RESULTADOS}

Con el propósito de implementar y validar el modelo propuesto, la estrategia es simulada en partidos en contra de un equipo oponente el cual utiliza una estrategia de equipo con 4 jugadores con roles constantes, siendo estos el portero, un defensa, un mediocampista y un delantero. El rol portero es similar al de la estrategia presentada. En la estrategia del oponente, el defensa intercepta el balón cuando este se encuentra en la zona ofensiva, y una vez lo tiene se lo envía al mediocampista, quien a su vez se lo envía nuevamente al delantero, este último trata de anotar un gol. Los comportamientos diseñados para el equipo oponente son similares a los de la estrategia propuesta; la diferencia fundamental consiste en que los roles en el oponente son 
constantes. No obstante, coordinación de equipo en la estrategia del oponente se presenta, incluyendo comportamientos cooperativos como la realización de pases.

Se realizaron un total de 200 juegos, cada uno de 10 minutos. La formación inicial en cada juego se cambia. En la Tabla III se presentan los resultados más relevantes de todos los juegos. Como se ha comentado, el equipo propio es el equipo con la estrategia propuesta, mientras que el equipo oponente es el equipo con la estrategia de roles constantes. Se puede observar que el promedio de goles por partido del equipo propio es significativamente mayor que el promedio de goles por partido del equipo oponente. También se puede apreciar que el equipo propio mantiene una mayor posesión del balón en todos los juegos. Adicionalmente, el porcentaje de juegos ganados por el equipo propio es considerablemente más alto que los empatados y los partidos perdidos.

TABLA III

RESULTADOS DE LOS 200 PARTIDOS

\begin{tabular}{lcc}
\hline Parámetros & Equipo Propio & Equipo Oponente \\
\hline Promedio Goles & 3.625 & 1.605 \\
Desviación estándar & 1.468 & 1.156 \\
\hline \% Posesión del balón & $65.49 \%$ & $34.51 \%$ \\
Desviación estándar & $10.6 \%$ & $10.6 \%$ \\
\hline \% Juegos ganados & $92.5 \%$ & $3.5 \%$ \\
\% Juegos empatados & $4 \%$ & $4 \%$ \\
\% Juegos perdidos & $3.5 \%$ & $92.5 \%$ \\
\hline
\end{tabular}

Como se aprecia también en la Tabla III, existen desviaciones estándar significativas tanto en el número de goles por partido de los dos equipos, como en el porcentaje de posesión del balón promedio en cada partido (la desviación estándar para este segundo caso es igual para los dos equipos, porque estos porcentajes son linealmente dependientes). Esto significa que existen variaciones entre el número de goles anotado por cada equipo entre los diferentes partidos, así como de la posesión del balón promedio por partido, entre los diferentes partidos. Esto se debe en principio a que el simulador Webots introduce un factor de aleatoriedad tanto en la detección de las texturas que permiten la localización de los robots, como en el ruido inducido en los sensores, esto con el propósito de inducir características estocásticas para realizar simulaciones más realistas [34].

Se realiza la prueba t-student para variables independientes con los resultados de los goles anotados por cada equipo en cada juego, para los 200 juegos. Obteniendo un valor $\mathrm{t}=15.29$, un valor crítico de $\mathrm{t}$ de dos colas de 1.966 , un valor $\mathrm{P}$ de dos colas de $2.1 \times 10^{-41}$ y teniendo en cuenta que se ha tomado un $\alpha=0.05$, se concluye que sí existen diferencias significativas en el número de goles anotados por el equipo con la estrategia propuesta frente al oponente el cual no presenta asignación dinámica de roles, observando que el equipo con la estrategia propuesta presenta una mejor adaptación a condiciones de ruido inducido en los sensores.

La Tabla IV presenta el porcentaje de tiempo en que cada táctica es seleccionada en todos los juegos y el porcentaje de tiempo que el balón es ubicado en cada zona, junto a sus desviaciones estándar D.E. DD corresponde a la táctica Defensiva, DA es la táctica contra ataque, OD corresponde a la táctica presión y $\mathrm{OA}$ es la táctica ataque. $\mathrm{DZ}$ corresponde a la zona defensiva, MZ es la zona media y OZ es la zona ofensiva.

TABLA IV

RESULTADOS DE LOS 200 PARTIDOS

\begin{tabular}{cccccccc}
\hline & \multicolumn{3}{c}{ \% de táctica } & \multicolumn{4}{c}{$\%$ Ubicación del balón } \\
\hline & DD & DA & OD & OA & DZ & MZ & OZ \\
Prom. & $19.5 \%$ & $35.8 \%$ & $15 \%$ & $29.7 \%$ & $35.2 \%$ & $20 \%$ & $44.8 \%$ \\
D.E & $6.75 \%$ & $5.95 \%$ & $5.07 \%$ & $9.89 \%$ & $5.93 \%$ & $4.9 \%$ & $8.51 \%$ \\
\hline
\end{tabular}

En promedio, la táctica contra ataque es la más seleccionada y la táctica presión ha sido la menos seleccionada. No obstante, es de resaltar que la táctica ataque es seleccionada en un porcentaje muy similar que la táctica contra ataque. Es de notar también que las tácticas contra ataque y ataque son más utilizadas porque el equipo propio mantiene la posesión del balón durante un porcentaje mayor de tiempo. La táctica contra ataque presenta un porcentaje levemente mayor, esto se debe porque esta táctica se activa estando el balón en las zonas defensivas y media, contrario a la táctica ataque que solamente puede ser activada cuando el balón está en la zona ofensiva. Es de destacar los valores en las desviaciones estándar tanto en el porcentaje de tiempo de selección de cada táctica como de la ubicación del balón, señalando variaciones significativas de los porcentajes de selección de táctica y ubicación del balón en algunos juegos.

Para apreciar mejor estos resultados, en la Fig. 10 presenta en diagrama de cajas el porcentaje de selección de cada táctica en cada juego y en la Fig. 11 se presenta igualmente en diagrama de cajas el porcentaje de ubicación del balón en cada zona por cada uno de los juegos. En general las táctica contra ataque $\mathrm{y}$ ataque (DA y OA) son seleccionadas más frecuentemente, confirmando los promedios presentados en la Tabla IV y como se comentó anteriormente, se debe a que el equipo propio presentó una mayor posesión del balón que el equipo oponente. Es bueno notar además que la táctica ataque (OA) muestra un porcentaje significativamente mayor de juego en muchos partidos, alcanzando en algunos casos valores cercanos al 70\%. No obstante, en otros juegos se observa que el porcentaje de tiempo en que la táctica contra ataque fue seleccionada es el mayor, siendo superior inclusive al $50 \%$. Estos resultados son coherentes con respecto a los porcentajes de tiempo de la ubicación del balón en las zonas, ya que en algunos juegos el balón puede estar hasta cerca del $70 \%$ del tiempo de juego en las zonas ofensivas, pero en otros juegos el balón se encuentra principalmente en las zonas defensiva o media, siendo consistente con los valores de desviación estándar presentados en la Tabla IV y debidos a la aleatoriedad inducida por el simulador.

En la Fig. 12 se presentan diagramas de caja de los porcentajes de tiempo en que cada rol es asignado a cada jugador en cada partido. En promedio los tres roles fueron asignados en porcentajes similares en cada jugador. No obstante se puede notar que en algunos partidos algún jugador tuvo asignado un rol específico en un mayor o menor 
porcentaje de tiempo. Una razón por la cual esta situación ocurre se debe a la formación inicial que como se explicó, se

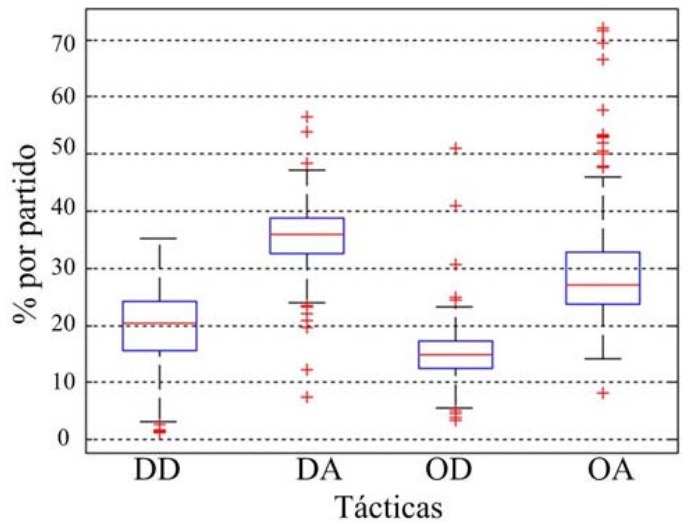

Figura 10. Porcentaje de selección de táctica en cada juego.

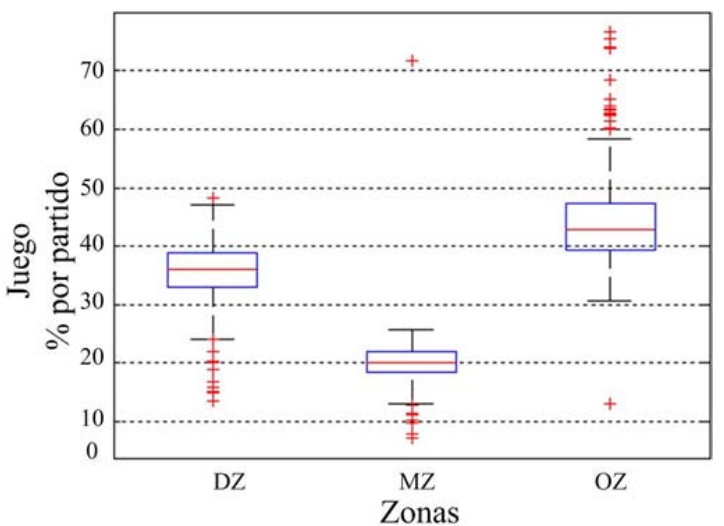

Figura 11. Porcentaje de ubicación del balón por zonas en cada juego.

cambiaba en cada juego, no obstante esta se mantenía durante el mismo juego cuando el partido se reiniciaba después que fuera anotado un gol, aunque es necesario resaltar qué esta situación se presentó en muy pocos partidos.

Muchos comportamientos han sido observados, por ejemplo la realización de pases entre el defensa y el mediocampista, o entre el mediocampista y el delantero, o la interceptación del balón cuando un oponente realiza un pase. El rol mediocampista ha sido muy importante en las tácticas ataque y presión, ya que se presentaron casos en los que el delantero dispara el balón pero este es desviado por un defensa oponente, logrando ser recuperado por el jugador que presentaba el rol de mediocampista.

\section{CONCLUCIONES}

En este artículo ha sido presentado un modelo de estrategia para fútbol de robots con asignación dinámica de roles realizada por un agente virtual llamado técnico, en un equipo con percepción global y control centralizado. Esta estrategia utiliza un número finito de tácticas, seleccionadas por una Máquina Jerárquica de Estado Finito y una vez el técnico virtual ha asignado los roles dependiendo de la ubicación del balón y los jugadores en el campo de juego, los robots con sus respectivos roles ejecutan diferentes comportamientos seleccionados por la Máquina Jerárquica de Estados Finitos, presentando diferentes comportamientos colaborativos. Una ventaja de este proceso es que no se requiere programación compleja. También es de resaltar que las arquitecturas jerárquicas de esta naturaleza son fácilmente expandibles a otras ligas con un número diferente de jugadores.

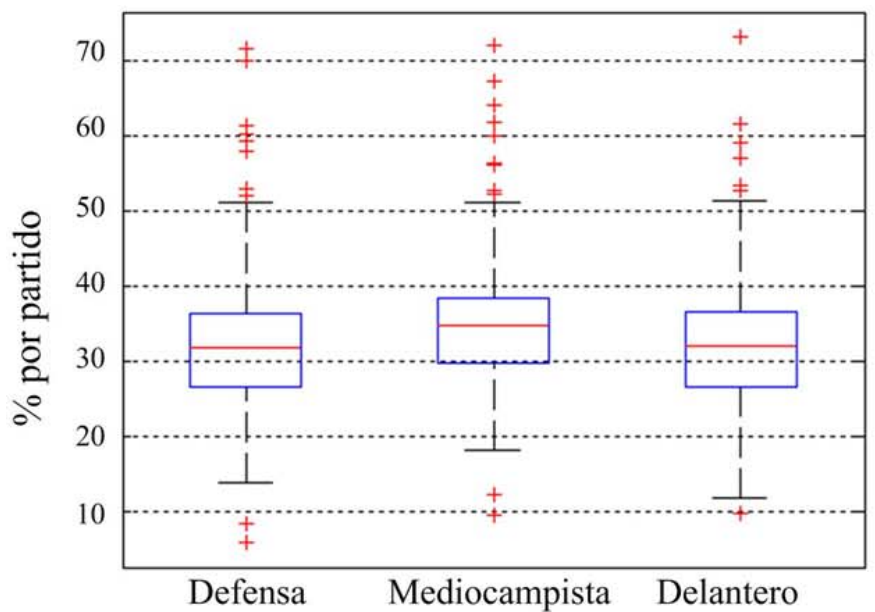

(a) Roles jugador 1

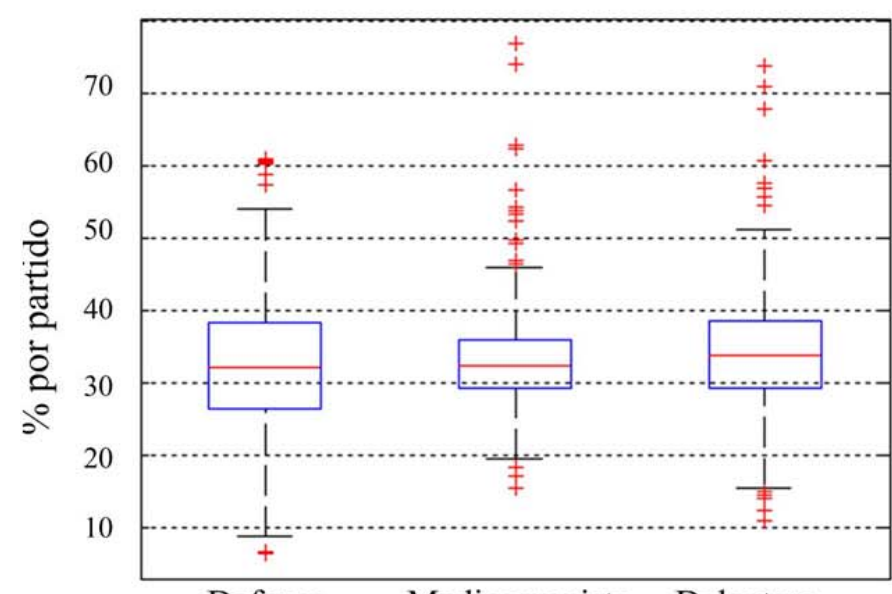

Defensa Mediocampista Delantero

(b) Roles jugador 2

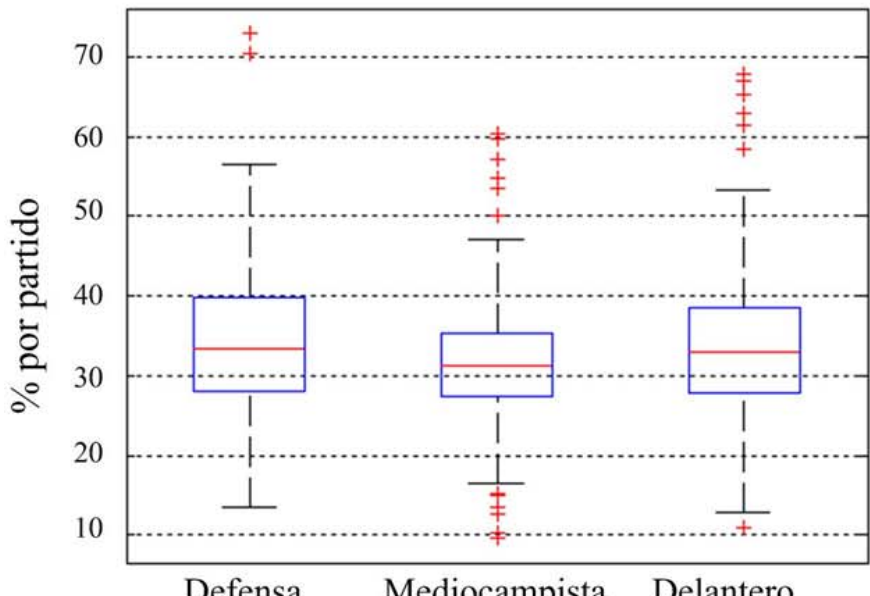

(c) Roles jugador 3

Figura 12. Porcentaje de selección de roles por jugador en cada juego. 
Para validar el modelo propuesto, se han realizado 200 partidos en simulación contra un equipo que incluye roles constantes. El número total de goles anotados por parte del equipo con la estrategia propuesta fue considerablemente mayor frente a los anotados por el oponente, así mismo ocurre con la posesión del balón. La ubicación del balón en el campo de juego fue mayoritariamente en la zona ofensiva, indicando que la estrategia propuesta presentaba jugadas principalmente de ataque sobre el equipo oponente con roles constantes. La selección dinámica de roles permitió comportamientos cooperativos tales como la realización de pases, la interceptación del balón en caso de pases del equipo oponente o recuperar la posesión del balón por parte de un jugador del equipo cuando su compañero la ha perdido, siempre manteniendo la continuidad del juego, ya que no se apreciaron comportamientos extraños o indebidos, como por ejemplo que un jugador abandone la posesión del balón para encargarse de un rol diferente, o que varios jugadores al mismo tiempo fueran a obstaculizarse mutuamente tratando de capturar el balón.

Las 4 tácticas presentadas en esta estrategia han sido seleccionadas en los 200 juegos. En la mayoría de los casos, se observó que las tácticas ofensivas (presión y ataque) fueron seleccionadas en mayores porcentajes, aunque esta diferencia porcentual no es muy grande. Adicionalmente el porcentaje de tiempo que los roles son asignados muestran que los jugadores ejecutan los 3 roles en similares proporciones. Este resultado indica que los roles son asignados de forma dinámica, dependiendo de las condiciones instantáneas del juego. Las mediciones obtenidas durante los juegos como por ejemplo el porcentaje de posesión del balón, número de goles anotados o el porcentaje de tiempo en que el balón se encuentra en la zona ofensiva, sugieren los beneficios de la estrategia propuesta con asignación dinámica de roles comparada con una estrategia de roles constantes. Otra ventaja de la estrategia propuesta es que presenta robustez en el caso de posible daño de un robot durante un juego: con un rol específico, un robot dañado podría no realizar importantes tareas, tales como disparar el balón a la portería oponente o bloquear a un contrincante.

Una forma de incrementar la efectividad de esta estrategia es mediante algoritmos de aprendizaje en los sistemas de toma de decisiones para la asignación de roles, así como utilizar algoritmos de aprendizaje en la ejecución de comportamientos con el propósito de mejorar acciones como la captura del balón, disparar el balón o la realización de pases. Otra posible línea de trabajo futuro, es usar algoritmos de predicción tanto en la ejecución de comportamientos, con el fin de tratar de predecir el lugar más adecuado para capturar el balón cuando no se tiene posesión del mismo o predecir el momento más oportuno para la transición de estados que permita la selección de un comportamiento nuevo, también se puede buscar predecir los comportamientos de los oponentes. Así mismo como trabajos futuros se propone integrar la arquitectura propuesta con sistemas de inferencia difusos, con el fin de mejorar la planeación de las trayectorias y el control de movimiento de los robots. También se explorará el uso de máquinas de estado no determinísticas y máquinas de estado probabilísticas para el diseño de estrategias de coordinación y sistemas de toma de decisiones, en ligas de fútbol de robots de percepción local y control distribuido.

\section{ANEXO}

En el anexo se presenta la estrategia propuesta, la cual se ejecuta desde el sistema de toma de decisiones. Las entradas del presente algoritmo corresponden a las coordenadas de todos los jugadores y el balón, y la salida del algoritmo corresponde a las señales de control de velocidad de las ruedas izquierda y derecha de cada uno de los robots (señal PWM).

\section{Estrategia jerárquica.}

Declarar funciones de comportamientos de jugadores;

Inicializar coordenadas de jugadores y el balón;

Detectar si hay posesión del balón;

zona_actual $=$ zona_ubicación_balón; $/ /$ zona defensiva $=1$, zona media $=2 y$ zona ofesiva $=3$

posesión_actual = valor posesión del balón; // 1 si hay posesión del balón, 0 si no

si (zona actual != zona anterior || posesión actual != posesión anterior) táctica = seleccionar nueva táctica; según (táctica) hacer:

caso 1: //Táctica defensa

Técnico asigna roles;

para $i=1$ hasta 3 ;

según (jugador_i) hacer:

caso 1: //Defensa

comportamiento ir al balón;

caso 2: //Mediocampista

comportamiento bloquear oponente;

case 3: //Delantero

comportamiento ir al centro;

fin según;

fin para;

caso 2: //Táctica Contra ataque

Técnico asigna roles;

para $i=1$ hasta 3 ;

según (jugador_i) hacer:

caso 1: //Defensa

comportamiento bloquear oponente;

caso 2: //Mediocampista

comportamiento balón a zona ofensiva;

case 3: //Delantero

comportamiento ir al centro;

fin según;

fin para;

caso 3: //Táctica Presión

Técnico asigna roles;

para $i=1$ hasta 3 ;

según (jugador_i) hacer:

caso 1: //Defensa

comportamiento ir zona defensiva;

caso 2: //Mediocampista

comportamiento esperar pase;

case 3: //Delantero

comportamiento ir al balón;

fin según;

fin para;

caso 4: //Táctica Ataque

Técnico asigna roles;

para $i=1$ hasta 3 ;

según (jugador_i) hacer:

caso 1: //Defensa

comportamiento ir zona defensiva;

caso 2: //Mediocampista

comportamiento esperar pase;

case 3: //Delantero

comportamiento enviar pase o disparar balón;

fin según;

fin para;

fin según; 
fin si;

zona_anterior $=$ zona_actual;

posesión anterior $=$ posesión actual;

Transmitir velocidades de los robots (rueda_izquierda, rueda_derecha);

\section{AGRADECIMIENTOS}

Jose Guillermo Guarnizo ha sido financiado por una beca del Departamento Administrativo de Ciencia, Tecnología e Innovación COLCIENCIAS, Colombia.

\section{REFERENCIAS}

[1] R. Ostos, F. Ramos, B. Cartillo and V. Félix, "Selection of Coordination Mechanisms in Intelligent Environments," Latin America Transactions, IEEE, vol. 13, n 9, pp. 3120-3126, September 2015.

[2] A. Farinelli, L. Iocchi and D. Nardi, "Multirobot systems: a classification focused on coordination," Systems, Man, and Cybernetics, Part B. Cybernetics, IEEE Transactions on, vol. 34, $n^{\circ} 5$, October 2004.

[3] J. M. Angel, G. E. Bravo C and F. De la Rosa R., "Electronics, Robotics and Automotive Mechanics Conference (CERMA), 2010," in Conceptualization of Human Soccer Concepts to Robotic Soccer Architecture, Morelos, 2010.

[4] N. Lau, L. S. Lopes, G. Corrente and N. Filipe, "Multi-robot team coordination through roles, positionings and coordinated procedures," in Intelligent Robots and Systems, 2009. IROS 2009. IEEE/RSJ International Conference on, St Louis, 2009.

[5] O. Zapata, G. Acosta and J. Jiménez, "Coordination Scheme and Behavior Fusion Based on Fuzzy Weighting Factors for a Mobile Robotic Navigation," Latin America Transactions, IEEE, vol. 13, $\mathrm{n}^{\circ}$ 1, pp. 18-26, January 2015.

[6] P. Stone y M. Veloso, "Task decomposition, dynamic role assignment, and low-bandwidth communication for real-time strategic teamwork," Journal of Artificial Intelligence, vol. 110, pp. 241-273, 1999.

[7] C. E. Agüero, V. Matellán, J. M. Cañas and V. M. Gómez, "SWITCH! Dynamic roles exchange among cooperative robots," in Second International Workshop on Multi-Agent Robotic Systems (MARS 2006), Setúbal, 2006.

[8] J. Testart, J. Ruiz del Solar, R. Schulz, P. Guerrero and R. PalmaAmestoy, "A Real-Time Hybrid Architecture for Biped Humanoids with Active Vision Mechanisms," Journal of Intelligent \& Robotic Systems, vol. $63, \mathrm{n}^{\circ} 2$, pp. 233-255, August 2011.

[9] C. Y. Low, N. Aziz, . M. Aldemir, M. Mellado, R. Dumitrescu and H. Anacker, "Principle Solution for Designing Collaborative Humanoid Soccer Robots," in International Symposium on Robotics and Intelligent Sensors 2012 (IRIS 2012), Sarawak, 2012.

[10] P. F. Palamar, V. A. Ziparo, L. Locchi, D. Nardi and P. Lima, "Teamwork Design Based on Petri Net Plans," in RoboCup 2008: Robot Soccer World Cup XII, 2009.

[11] J. Wang, T. Wang, X. Wang and X. Meng, "Multi-robot decision making based on coordination graphs," in Mechatronics and Automation, 2009. ICMA 2009. International Conference on, Changchun, 2009.

[12] G. Kontes and M. G. Lagoudakis, "Coordinated Team Play in the FourLegged RoboCup League," in Tools with Artificial Intelligence, 2007. ICTAI 2007. 19th IEEE International Conference on, Patras, 2007.

[13] J. Ibarra Zannatha, L. Figueroa Medina, R. Cisneros Limón and P. Mejía Álvarez, "Behavior control for a humanoid soccer player using Webots," in Electrical Communications and Computers (CONIELECOMP), 2011 21 st International Conference on, San Andres Cholula, 2011.

[14] P. Cardoso, L. Molina and E. O. Freire, "A Methodology to Designing Strategies for Robot Soccer Based on Discrete Event Systems Formalism," in Robotics Symposium and Latin American Robotics Symposium (SBR-LARS), 2012 Brazilian, Fortaleza, 2012.

[15] M. Yang and Y. Jia, "Action Utility Prediction and Role Task Allocation in robot soccer system," in Control Automation Robotics \& Vision (ICARCV), 2012 12th International Conference on, Guangzhou, 2012.

[16] Y. Lou, B. Chen and H. Shi, "Decision making model based on state assessment and hierarchical FSM in robot soccer," in Automatic Control and Artificial Intelligence (ACAI 2012), International Conference on, Xiamen, 2012.

[17] J. Wu, E. Ochodkova, J. Martinovic, V. Snásel and A. Abraham, "Analysis of loop strategies in robot soccer game," de Intelligent Systems Design and Applications (ISDA), 2011 11th International Conference on, Cordoba, 2011.

[18] H. Shi, L. Xu, L. Zhang, W. Pan and G. Xu, "Research on self-adaptive decision-making mechanism for competition strategies in robot soccer," Frontiers of Computer Science, vol. 9, nº 3, pp. 485-494, June 2015.

[19] K. S. Hwang, S. W. Tan and C. C. Chen, "Cooperative strategy based on adaptive Q-learning for robot soccer systems," Fuzzy Systems, IEEE Transactions on, vol. 12, $\mathrm{n}^{\mathrm{o}}$ 4, pp. 569-576, August 2004.

[20] V. Hilaire, A. Koukam and S. Rodriguez, "An Adaptative Agent Architecture for Holonic Multi-Agent Systems," ACM Transactions on Autonomous and Adaptive Systems, vol. 3, n 1, pp. 2-24, March 2008.

[21] Y. T. Wang and C. H. Chen, "Design of an Action Select Mechanism for Soccer Robot Systems Using Artificial Immune Network," Tamkang Journal of Science and Engineering, vol. 11, n 4, pp. 415-424, 2008.

[22] G. Zuo, S. Zhang, G. Zhong and R. Sun, "Two-agent collaborative shooting method using BP neural network," in Intelligent Computing and Intelligent Systems (ICIS), 2010 IEEE International Conference on, Xiamen, 2010.

[23] S. L. Wu, Y. R. Liou, W. H. Lin and M. H. Wu, "A Multi-agent Algorithm for Robot Soccer Games in Fira Simulation League," in FIRA RoboWorld Congress, San Francisco, 2007.

[24] B. Fu, P. X. Zhang and C. F. Wang, "A Cooperation Strategy for Shooting in Robot Soccer Competition Based on the Multi-Suppose Tree," in 2012 International Workshop on Information and Electronics Engineering, Harbin, 2012.

[25] J. Lee, D. Ji, W. Lee, G. Kang and M. G. Joo, "A Tactics for Robot Soccer with Fuzzy Logic Mediator," in International Conference, CIS 2005, Xi'an, China, December 15-19, 2005, Proceedings Part I, XIan, 2005.

[26] K. S. Hwang, S. W. Tan and C. C. Chen, "Cooperative strategy based on adaptive Q-learning for robot soccer systems," Fuzzy Systems, IEEE Transactions on, vol. 12, no 4, pp. 569-576, August 2004.

[27] C. J. Wu and T. L. Lee, "A Fuzzy Mechanism for Action Selection of Soccer Robots," Journal of Intelligent \& Robotic Systems, vol. 39, $\mathrm{n}^{\circ} 1$, pp. 57-70, January 2004.

[28] D. Gu and H. Hu, "Integration of Coordination Architecture and Behavior Fuzzy Learning in Quadruped Walking Robots," Systems, Man and Cybernetics, Part C: Applications and Reviews, IEEE Transactions on, vol. 37, $\mathrm{n}^{\circ} 4$, pp. 670-681, 2007.

[29] G. C. Luh, C. Y. Wu and W. W. Liu, "Artificial Immune System based Cooperative Strategies for Robot Soccer Competition," in Strategic Technology, The 1st International Forum on, Ulsan, 2006.

[30] K. Jolly, R. Sreerama Kumar and R. Vijayakumar, "Intelligent task planning and action selection of a mobile robot in a multi-agent system through a fuzzy neural network approach," Engineering Applications of Artificial Intelligence, vol. 23, $\mathrm{n}^{\circ}$ 6, pp. 923-933, September 2010.

[31] R. R. P. Vicerra, K. K. A. David, A. R. de la Cruz, E. A. Roxas, K. B. C. Simbulan, A. A. Bandala and E. P. Dadios, "A multiple level MIMO fuzzy logic based intelligence for multiple agent cooperative robot system," in TENCON 2015 - 2015 IEEE Region 10 Conference, Macao, 2015.

[32] T. Srinivasan, S. Harini, K. Kavitha and P. Sukumar, "SVMRobosoc: A Novel Approach Towards Planning Strategy for Robotic Soccer Using Hierarchical Intelligence Systems," in Seventh International Conference on Intelligent Systems Design and Applications (ISDA 2007), Rio de Janeiro, 2007.

[33] B. Chen, A. Zhang and L. Cao, "Autonomous intelligent decisionmaking system based on Bayesian SOM neural network for robot soccer," Neurocomputing, vol. 128, pp. 447-458, 2014.

[34] B. Magyar, Z. Forhecz and P. Korondi, "Developing an efficient mobile robot control algorithm in the Webots simulation environment," in Industrial Technology, 2003 IEEE International Conference on, 2003. 


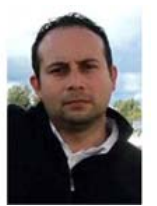

Jose Guillermo Guarnizo is Electronic Engineer of the University Francisco Jose de Caldas of Bogota Colombia in 2006, Master in Industrial Automation of the National University of Colombia in 2009, and Ph.D in Automatic, Robotics, and Industrial Informatics in Universitat Politècnica de València 2016. José Guillermo belongs to the Laboratory of Alternative Source of Energy LIFAE of the University Francisco José de Caldas of Bogota Colombia. His main research interests are in the field of robotics, control systems and intelligence systems.

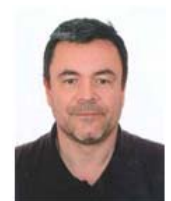

Martin Mellado received the B.Eng. in Computer Science from Universitat Politècnica de València (UPV), Spain in 1987, the MSc degree in Computer Aided Engineering from the School of Mechanical Engineering, Cranfield Institute of Technology, UK in 1989 and the PhD degree in Computer Engineering in UPV in 1996. Nowadays he is a Professor and develops his research in the Instituto de Automática e Informática Industrial in UPV. His main research interests are in the field of food inspection and manipulation with robots, leading more than $12 \mathrm{R} \& \mathrm{D}$ projects in this field. 